\title{
Computing LF-Metric Dimension of Generalized Gear Networks
}

\author{
Hassan Zafar, ${ }^{1}$ Muhammad Javaid $\mathbb{D}^{1},{ }^{1}$ and Ebenezer Bonyah $\mathbb{D}^{2}$ \\ ${ }^{1}$ Department of Mathematics, School of Science, University of Management and Technology, Lahore 54770, Pakistan \\ ${ }^{2}$ Department of Mathematics Education, Akenten Appiah-Menka University of Skills Training and Entrepreneurial Development, \\ Kumasi 00233, Ghana \\ Correspondence should be addressed to Ebenezer Bonyah; ebbonya@gmail.com
}

Received 10 September 2021; Accepted 12 November 2021; Published 30 November 2021

Academic Editor: Muhammad Faisal Nadeem

Copyright (C) 2021 Hassan Zafar et al. This is an open access article distributed under the Creative Commons Attribution License, which permits unrestricted use, distribution, and reproduction in any medium, provided the original work is properly cited.

\begin{abstract}
The parameter of distance in the theory of networks plays a key role to study the different structural properties of the understudy networks or graphs such as symmetry, assortative, connectivity, and clustering. For the purpose, with the help of the parameter of distance, various types of metric dimensions have been defined to find the locations of machines (or robots) with respect to the minimum consumption of time, the shortest distance among the destinations, and the lesser number of utilized nodes as places of the objects. In this article, the latest derived form of metric dimension called as LF-metric dimension is studied, and various results for the generalized gear networks are obtained in the form of exact values and sharp bounds under certain conditions. The LFmetric dimension of some particular cases of generalized gear networks (called as generalized wheel networks) is also illustrated. Moreover, the bounded and unboundedness of the LF-metric dimension for all obtained results is also presented.
\end{abstract}

\section{Introduction}

Slater defined the idea of the resolving sets for a connected network to find the reference or location number for a connected network [1]. Harary and Melter studied the same concept of location number with the different term called metric dimension (MD) for connected networks [2]. Melter and Tomescu investigated metric basis in digital geometry those often used in city block distance [3], and Slater studied the dominating and reference sets in a network [4]. Garey and Johnson proved that finding MD of all the connected networks in general form is an NP complete problem [5]. Moreover, Kelenca et al. defined the idea of edge MD, they showed that computing edge MD is NP-complete problem [6], and the directed distance dimension of oriented networks is computed by Chattrand et al. [7]. For further studies of MD of Caylay, mobius ladders, lexicographic product, and Toeplitz networks, we refer to [8-11].

The concepts of various metric dimensions used in engineering are robot navigation, image processing, and pattern recognition [12]. It is used to solve problems involving percolation in hierarchical lattice [13] and to study the structural properties of chemical compounds. More importantly, Chartrand et al. used the concept of MD to solve an integer programming problem (IPP) [14]. The generalized Jahangir network $J_{m, k}$ is defined by Tomescu and Javaid [15], and they also computed MD for $J_{2 n}$. Furthermore, MD of generalized certain gear networks $J_{2 n, m}$ and $J_{3 n}$ was computed by Imran et al. [16].

Recently, Currie and Oellermann defined the concept of fractional metric dimension (FMD) to improve the solution of the linear relaxation of the IPP [17]. Fehr et al. used FMD to obtain an optimal solution of IPP [18]. Arumugam and Matthew calculated the exact values of FMD of some connected networks [19]. The bounds and exact values of FMD of vertex-transitive and distance-regular networks are computed by Feng et al. [20]. Saputro et al. computed the FMD of comb product of connected networks [21]. For further studies of FMD for hierarchical product, trees, and unicyclic and permutation networks, see [22-24]. Raza et al. computed the bounds of FMD of metal organic frameworks [25]. The FMD of generalized Jahangir network $J_{m, k}$ for $m=5$ is computed by Liu et al. [26].

The idea of LF- metric dimension is defined by Aisyah et al., and they also computed exact values for LF- metric dimension for the corona product of connected networks [27]. Liu et al. 
[28] studied the LF-metric dimension of triangular circular, quadrangular circular, and pentagonal circular ladders. Javaid et al. established the criteria to compute the upper bounds of LFmetric dimension of connected networks and demonstrated the main results by using LF-metric dimension such as wheel-related networks, flowers, and antiweb gear networks [29]. Recently, Javaid et al. improved the lower bound of LF-metric dimension from unity and established the sharp bounds and computed the exact values of LFMD of some prism-related networks [30].

In this article, we have computed LF-metric dimension of generalized gear networks in the form of exact values and sharp bounds. Furthermore, Sections 2 and 3 consist of preliminaries and LRN sets of $J_{m, k}$, respectively. In Section 4, we have computed LF-metric dimension of generalized gear networks, and Section 5 represents the conclusion and limiting values of LF-metric dimension.

\section{Preliminaries}

Let $G=(V(G), E(G))$ be a network with $V(G)$ and $E(G) \subseteq V(G) \times V(G)$ as vertex and edge set, respectively. A walk is a sequence $u_{o}, e_{1}, u_{1}, \ldots, u_{m-1}, e_{m}, u_{m}$ of vertices and edges such that the edge $e_{i}$ has end points $u_{i-1}$ and $u_{i}$ for $1 \leq i \leq m$. A path between two vertices $u$ and $v$ is called a walk if the repetition of vertices does not exist [31]. For any two vertices $u, v \in V(G)$, the distance $d(u, v)$ is the length of the shortest path between them in $G$. A network is said to be connected if there exists a path between any pair of vertices. A vertex $x \in V(G)$ is said to resolve a pair $\{a, b\} \subseteq V(G)$ if $d(x, a) \neq d(x, b)$. Let $S=\left\{v_{1}, v_{2}, v_{3}, \ldots, v_{m}\right\} \subseteq V(G)$ be an ordered set and $x \in V(G)$, then the $m$ tuple representation of $x$ with respect to $S$ is $d(x \mid S)=\left(\left(x, v_{1}\right)\right.$, $\left.\left(x, v_{2}\right),\left(x, v_{3}\right), \ldots,\left(x, v_{m}\right)\right)$. If the distinct vertices of $G$ have distinct representations with respect to $S$, then $S$ is called resolving (locating) set. The resolving set with minimum cardinality is called the metric basis of $G$, and the cardinality of metric basis is called metric dimension of $G$ defined as

$$
\operatorname{dim}(G)=\min \{|S|: S \text { is the resolving set of } G\} .
$$

For an edge $a b \in E(G)$, the local resolving neighbourhood set of $G$ is $\operatorname{LR}(a b)=\{z \in V(G): d(z, a) \neq d(z, b)\}$. A real-valued function $f: V(G) \longrightarrow[0,1]$ such that $f(\operatorname{LR}(a b)) \geq 1$ for each $\operatorname{LR}(a b)$ is called a local resolving function (LRF) of $G$, where $f(\operatorname{LR}(a b))=\sum_{x \in \operatorname{LR}(a b)} f(x)$. A LRF $f$ is called minimal if there exists a function $g: V(G) \longrightarrow[0,1]$ such that $g \leq f$ and $g(x) \neq f(x)$ for at least one $x \in G$ that is not LRF of G. LF-metric dimension of $G$ is donated by $\operatorname{dim}_{l f}(G)$ and defined as

$$
\operatorname{dim}_{l f}(G)=\min \{|f|: f \text { is minimal local resolving function of } G\} \text {. }
$$

Now, we present some important results frequently used in this paper.

Theorem 1 (see [29]). Let $G$ be a connected network. If $G$ is bipartite network, then $\operatorname{dim}_{l f}(G)=1$.
Theorem 2 (see [29]). Let $G$ be a connected network and $L R(e)$ be a LRN set for some $e \in E(G)$. If $|L R(e) \cap A| \geq \alpha, \forall e \in E(G)$, then

$$
1 \leq \operatorname{dim}_{l f}(G) \leq \frac{|A|}{\alpha},
$$

where $A=\bigcup\{L R(e):|L R(e)|=\alpha\}, \alpha=\min \{|L R(e)|: e \in E$ $(G)\}$, and $2 \leq \alpha \leq|V(G)|$.

Theorem 3 (see [30]). Let $G$ be a connected network and $L R(e)$ be the LRN set. Then,

$$
\begin{aligned}
\frac{|V(G)|}{\lambda} & \leq \operatorname{dim}_{l f}(G), \\
\lambda & =\max \{|\operatorname{LR}(e)|: e \in E(G)\}, \\
2 & \leq \lambda \leq|V(G)| .
\end{aligned}
$$

Corollary 1 (see [30]). Let $G=(V(G), E(G))$ be a connected network, $L R(e)$ be $L R N$ of $e \in E(G)$, $\lambda=\max \{|L R(e)|: e \in E(G)\}, \quad \alpha=\min \{|L R(e)|: e \in E(G)\}$, and $X=\bigcup\{L R(e):|L R(e)|=\alpha\}$. If $\alpha=\lambda$ and $X=V(G)$, then

$$
\operatorname{dim}_{l f}(G)=\frac{|V(G)|}{\lambda} .
$$

Proposition 1 (see [28]). Let $G=(V(G), E(G))$ be a connected network $X=\bigcup\{L R(e):|L R(e)|=2\}$. If $|L R(e) \cap X| \geq 2$ for all $e \in E(G)$, then $\operatorname{dim}_{\text {If }}=|X| / 2$.

Now, we define generalized gear network as follows.

Definition 1. Generalized gear network sometimes known as bipartite wheel network is obtained from wheel network. Let $J_{m, k}$ be the generalized gear network of order $m(k+1)+1$, where $m \geq 3$ and $k \geq 1$. There are three type of vertices in $J_{m, k}$ such as major vertices $a_{i}$, minor vertices $b_{k}^{i}$, where $1 \leq i \leq m$ and a central vertex $b$. Moreover, $E\left(J_{m, k}\right)=\left\{a_{i} b_{k}^{i}, 1 \leq i \leq m\right\}$ $\bigcup\left\{b_{k}^{i} b_{k}^{i+1}, 1 \leq i \leq m\right\} \bigcup\left\{a_{i} b, 1 \leq i \leq m\right\}$. Central vertex is adjacent to $m$ major vertices and contains an outer cycle $c_{m(k+1)}$ as shown in Figure 1. For more details of $J_{m, k}$, see [16].

\section{LRN Sets of the Generalized Gear Network $\left(J_{m, k}\right)$}

The resolving neighbourhood sets for each pair of adjacent vertices are classified.

Lemma 1. Let $J_{m, 0}$ with $m \geq 6$ be a generalized gear network, where $\left|V\left(J_{m, 0}\right)\right|=m+1$. Then, for $1 \leq i \leq m$, we have

(a) $\left|\operatorname{LR}\left(e_{i}\right)\right|=\left|\operatorname{LR}\left(a_{i} a_{i+1}\right)\right|=4$ and $\left|\bigcup_{i=1}^{m} L R\left(e_{i}\right)\right|=m$

(b) $\left|L R\left(e_{i}\right)\right| \leq|L R(x)|$, and $\left|L R(x) \cap \bigcup_{i=1}^{m} L R\left(e_{i}\right)\right| \geq \mid L R$ $\left(e_{i}\right) \mid \forall x \in E\left(J_{m, 0}\right)$ 


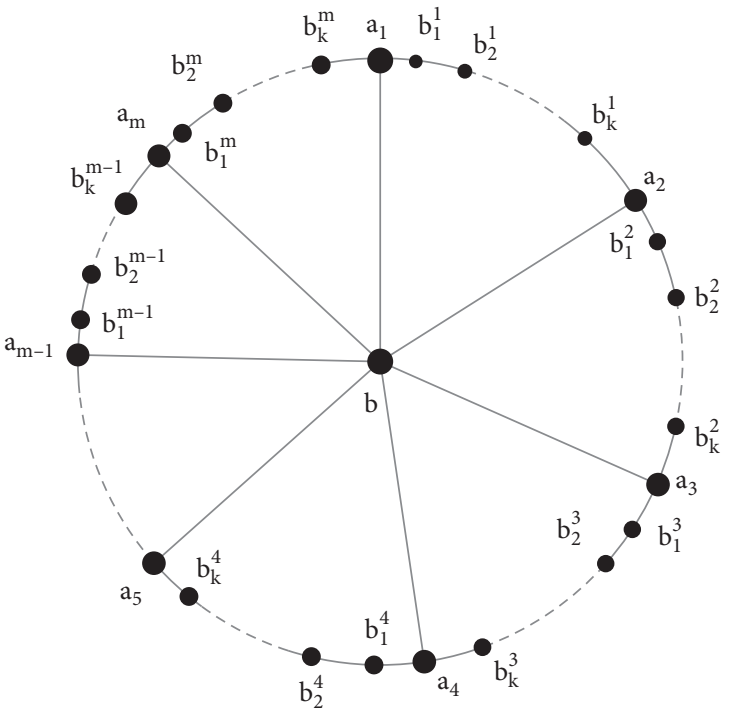

Figure 1: Generalized gear network $J_{m, k}$.

Proof. Assume that $a_{i}$ (major) vertices for $1 \leq i \leq m$ and $b$ (centre) vertex of $J_{(m, 0)}$ such that $a_{m}=a_{-1}$ and $a_{m+1}=a_{1}$.

(a) Consider $\operatorname{LR}\left(e_{i}\right)=\operatorname{LR}\left(a_{i} a_{i+1}\right)=\left\{a_{i}, a_{i+1}, a_{i+2}, a_{i-1}\right\}$, this implies that $\left|\operatorname{LR}\left(e_{i}\right)\right|=4$, where $1 \leq i \leq m$. Furthermore, $\left|\bigcup_{i=1}^{m} \operatorname{LR}\left(e_{i}\right)\right|=m$.

(b) Consider $\operatorname{LR}\left(a_{i} b\right)=V\left(J_{m, 0}\right)-\left\{a_{i-1}, a_{i+1}\right\}$, therefore $\left|\operatorname{LR}\left(a_{i} b\right)\right|=m-1$. Moreover, $\left|\operatorname{LR}\left(a_{i} b\right)\right|>\left|\operatorname{LR}\left(e_{i}\right)\right|$ and $\left|\operatorname{LR}(x) \cap \bigcup_{i=1}^{m} \operatorname{LR}\left(e_{i}\right)\right| \geq\left|\operatorname{LR}\left(e_{i}\right)\right|, \forall x \in E\left(J_{m, 0}\right)$.

Lemma 2. Let $J_{m, k}$ with $m \geq 4$ be a generalized gear network, where $m \cong 0(\bmod 2)$, and $k \cong 0(\bmod 2)$. Then, for $1 \leq i \leq m$, we have

(a) $\left|\operatorname{LR}\left(e_{i}\right)\right|=\left|\operatorname{LR}\left(b_{(k / 2)}^{i} b_{(k / 2)+1}^{i}\right)\right|=2 k+4$ and $\mid \bigcup_{i=1}^{m} L R$ $\left(e_{i}\right)|=| V\left(J_{m, k}\right) \mid-1$

(b) $\left|L R\left(e_{i}\right)\right| \leq|L R(x)|$ and $\left|L R(x) \cap \bigcup_{i=1}^{m} L R\left(e_{i}\right)\right| \geq \mid L R$ $\left(e_{i}\right) \mid, \forall x \in E\left(J_{m, k}\right)$

Proof. Assume that $a_{i}$ (major), $b$ (center), and $b_{k}^{i}$ be a minor vertex, respectively, where $1 \leq i \leq k$ and $(i+P) \cong P(\bmod k)$. Now, we have,

(a) Consider $\quad \operatorname{LR}\left(e_{i}\right)=\operatorname{LR} \quad\left(b_{(k / 2)}^{i} b_{(k / 2)+1}^{i}\right)=$ $\left\{b_{(k / 2)}^{i}, b_{(k / 2)+1}^{i}, \ldots, b_{(k / 2)+1}^{i+1}\right\} \bigcup\left\{\left(b_{(k / 2)}^{m}, b_{(k / 2)+i}^{i}, \ldots\right.\right.$, $\left.\left.b_{(k / 2)-1}^{i}\right)\right\}, \quad\left|\operatorname{LR}\left(e_{i}\right)\right|=2 k+4$ and $\left|\bigcup_{i=1}^{m} \operatorname{LR}\left(e_{i}\right)\right|=$ $\left|V\left(J_{m, k}\right)\right|-1$

(b) LRNs other than $\operatorname{LR}\left(e_{i}\right) \operatorname{areLR}\left(a_{i} b\right)=V\left(J_{m, k}\right)-$ $\left\{b_{(k+2) / 2}^{i}, b_{k / 2}^{i+k+1}\right\}, \operatorname{LR}\left(a_{i} b_{1}^{i}\right)=V\left(J_{m, k}\right)-\left\{b_{(k+4) / 2}^{i}\right\}, \operatorname{LR}$ $\left(a_{i} b_{k}\right)=V\left(J_{m, k}\right)-\left\{b_{(k / 2)-1}^{i}\right\}, \quad \operatorname{LR}\left(b_{1}^{i} b_{2}^{i}\right)=V\left(J_{m, k}\right)-$ $\left\{b_{(k+2) / 2}^{m}, b_{((k+2) / 2)+1}^{m}, \ldots, a_{i}\right\}, \quad$ for $(k=2) \operatorname{LR}\left(b_{1}^{i} b_{2}^{i}\right)$ $=V\left(J_{m, 2}\right)-\left\{a_{i+1}, b_{1}^{i+1}, b_{k}^{i+1}\right\}$

The cardinality of each LRN set is shown in Table 1 .
TABLE 1: Cardinality of each LRN set.

\begin{tabular}{lc}
\hline LRN set & Cardinality \\
\hline $\operatorname{LR}\left(a_{i} b\right)$ & $\left|V\left(J_{m, k}\right)\right|-2>\left|\operatorname{LR}\left(e_{i}\right)\right|$ \\
$\operatorname{LR}\left(a_{i} b_{1}^{i}\right)$ & $\left|V\left(J_{m, k}\right)\right|-4>\left|\operatorname{LR}\left(e_{i}\right)\right|$ \\
$\operatorname{LR}\left(a_{i} b_{1}^{i}\right)(k=2)$ & $\left|V\left(J_{m, k}\right)\right|-3>\left|\operatorname{LR}\left(e_{i}\right)\right|$ \\
$\operatorname{LR}\left(a_{i} b_{k}\right)$ & $\left|V\left(J_{m, k}\right)\right|-1>\left|\operatorname{LR}\left(e_{i}\right)\right|$ \\
\hline
\end{tabular}

From Table 1, it is clear that $\left|\operatorname{LR}\left(e_{i}\right)\right| \leq|\operatorname{LR}(x)|, \forall x \in$ $E\left(J_{m, k}\right)$. Furthermore, $\left|\bigcup_{i=1}^{m} \operatorname{LR}\left(e_{i}\right)\right|=\left|V\left(J_{m, k}\right)\right|-1$; therefore, $\left|\operatorname{LR}(x) \cap \bigcup_{i=1}^{m} \operatorname{LR}\left(e_{i}\right)\right| \geq\left|\operatorname{LR}\left(e_{i}\right)\right| \forall x \in E\left(J_{m, k}\right)$.

Lemma 3. Let $\left(J_{m, k}\right)$ be a generalized gear network, where $k \cong 0(\bmod 2)$ and $m \cong 1(\bmod 2)$. Then, for $1 \leq i \leq m$, we have

(a) $\left|L R\left(e_{i}\right)\right|=\left|L R\left(b_{(k / 2)}^{i} b_{(k / 2)+1}^{i}\right)\right|=2 k+4$ and $\mid \bigcup_{i=1}^{m} L R$ $\left(e_{i}\right)|=| V\left(J_{m, k}\right) \mid-1$

(b) $\left|L R\left(e_{i}\right)\right| \leq|L R(x)|$ and $\left|L R(x) \cap \bigcup_{i=1}^{m} L R\left(e_{i}\right)\right| \geq \mid L R$ $\left(e_{i}\right) \mid, \forall x \in E\left(J_{m, k}\right)$

Proof. Assume that $a_{i}$ (major), $b$ (center) and $b_{k}^{i}$ be the $\operatorname{minor}$ vertex, for $1 \leq i \leq m,(m+1) \bmod m=1$. Now, we have

(a) $\operatorname{LR}\left(e_{i}\right)=\left\{\operatorname{LR}\left(b_{(k / 2)}^{i} b_{(k / 2)+1}^{i}\right)\right\}=\left\{\left(b_{(k / 2)}^{i}, b_{(k / 2)+1}^{i}, \ldots\right.\right.$, $\left.b_{(k / 2)+1}^{i+1}\right\} \bigcup\left\{\left(b_{(k / 2)}^{m}, b_{(k / 2)+i}^{m}, \ldots, b_{(k / 2)-1}^{i}\right)\right\},\left|\operatorname{LR}\left(e_{i}\right)\right|=$ $2 k+4$ and $\bigcup_{i=1}^{m} \operatorname{LR}\left(e_{i}\right)=\left|V\left(J_{m, k}\right)\right|-1$.

(b) LRNs other than $\operatorname{LR}\left(e_{i}\right)$ are $\operatorname{LR}\left(a_{i} b_{k}^{i}\right)=V\left(J_{m, 4}\right)$ $-\left\{b_{k}^{i}\right\}, \operatorname{LR}\left(a_{i} b_{k}^{i}\right)$ for $(k \geq 6)=V\left(J_{m, k}\right)-\left\{b_{(k / 2)+1}^{i}\right\}$, $\operatorname{LR}\left(a_{i} b\right)=V\left(J_{m, k}\right)-\left\{\left(b_{(k / 2)+1}^{i}, b_{(k / 2)}^{m}\right\}, \quad \operatorname{LR}\left(b_{1}^{i} b_{2}^{i}\right)=\right.$ $V\left(J_{m, 4}\right)-\left\{a_{i+1}, b_{1}^{i+1}, b_{2}^{i+1}\right\}, \quad \operatorname{LR}\left(b_{1}^{i} b_{2}^{i}\right)(k \geq 6)=V$ $\left(J_{m, k}\right)-\left\{b_{(k+6) / 2}^{i}\right\}, \quad \operatorname{LR}\left(b_{3}^{i} b_{4}^{i}\right)=V\left(J_{m, 4}\right)-\left\{a_{i-1}, b_{4}^{i-1}\right.$, $\left.b_{4}^{i-1}\right\}, \quad \operatorname{LR}\left(b_{k-1}^{i} b_{k}^{i}\right)=V\left(J_{m, k}\right)-\left\{b_{(k-4) / 2}^{i}\right\} \operatorname{LR}\left(b_{k-2}^{i} b_{k}\right.$ $\left.-1^{i}\right)=V\left(J_{m, k}\right)-\left\{b_{(k-6) / 2}^{i}\right\}$, and $\operatorname{LR}\left(b_{2}^{i} b_{3}^{i}\right)=V\left(J_{m, k}\right)$ $-\left\{b_{(k+8) / 2}^{i}\right\}$.

The cardinality of each LRN set is shown in Table 2.

From Table 2, it is clear that $\left|\operatorname{LR}\left(e_{i}\right)\right| \leq|\operatorname{LR}(x)|, \forall x \in$ $E\left(J_{m, k}\right)$. Furthermore, $\quad\left|\bigcup_{i=1}^{m} \operatorname{LR}\left(e_{i}\right)\right|=\left|V\left(J_{m, k}\right)\right|-1$. Therefore,

$\left|\operatorname{LR}(x) \cap \bigcup_{i=1}^{m} \operatorname{LR}\left(e_{i}\right)\right| \geq\left|\operatorname{LR}\left(e_{i}\right)\right| \forall x \in E\left(J_{m, k}\right)$.

\section{LFMD of Generalized Gear Networks $\left(J_{m, k}\right)$}

In this section, we compute LF-metric dimension of generalized gear network in the form of exact values and bounds under certain conditions.

4.1. For $k=0$ and $m \geq 3$. In this particular section, we determine the LF-metric dimension of the generalized wheel network, which is the special case of the generalized gear network. For $k=0$, let $A=\left\{a_{1}, a_{2}, a_{3}, a_{4}, \ldots, a_{m}\right\}$ be the set of outer vertices and $b$ is the central vertex of $J_{m, 0}$.

Theorem 4. Let $J_{3,0}$ be a generalized gear network, then $\operatorname{dim}_{l f}\left(J_{3,0}\right)=2$. 
TABLE 2: Cardinality of each LRN set.

\begin{tabular}{lc}
\hline LRN set & Cardinality \\
\hline $\operatorname{LR}\left(a_{i} b_{k}^{i}\right)$ & $\left|V\left(J_{m, k}\right)\right|-1>\left|\operatorname{LR}\left(e_{i}\right)\right|$ \\
$\operatorname{LR}\left(a_{i} b\right)$ & $\left|V\left(J_{m, k}\right)\right|-1>\left|\operatorname{LR}\left(e_{i}\right)\right|$ \\
$\operatorname{LR}\left(b_{1}^{i} b_{2}^{i}\right)(k=4)$ & $\left|V\left(J_{m, 4}\right)\right|-3>\left|\operatorname{LR}\left(e_{i}\right)\right|$ \\
$\operatorname{LR}\left(b_{1}^{i} b_{2}^{i}\right) k \geq 6$ & $\left|V\left(J_{m, k}\right)\right|-3>\left|\operatorname{LR}\left(e_{i}\right)\right|$ \\
$\operatorname{LR}\left(a_{i} b_{1}^{i}\right)(k=2)$ & $\left|V\left(J_{m, k}\right)\right|-3>\left|\operatorname{LR}\left(e_{i}\right)\right|$ \\
$\operatorname{LR}\left(a_{i} b_{k}\right)$ & $\left|V\left(J_{m, k}\right)\right|-1>\left|\operatorname{LR}\left(e_{i}\right)\right|$ \\
$\operatorname{LR}\left(b_{3}^{i} b_{4}^{i}\right)(k=4)$ & $\left|V\left(J_{m, 4}\right)\right|-3>\left|\operatorname{LR}\left(e_{i}\right)\right|$ \\
$\operatorname{LR}\left(b_{k-1}^{i} b_{k}^{i}\right)$ & $\left|V\left(J_{m, k}\right)\right|-1>\left|\operatorname{LR}\left(e_{i}\right)\right|$ \\
$\operatorname{LR}\left(b_{k-2}^{i} b_{k-1}^{i}\right)$ & $\left|V\left(J_{m, k}\right)\right|-1>\left|\operatorname{LR}\left(e_{i}\right)\right|$ \\
\hline
\end{tabular}

Proof. For $m=3$ and $k=0$. The LRN sets are given as follows.

The LRN sets of major vertices to central vertex are as follows:

$$
\begin{aligned}
& \operatorname{LR}_{1}=\operatorname{LR}\left(a_{1} b\right)=\left\{a_{1}, b\right\} \\
& \operatorname{LR}_{2}=\operatorname{LR}\left(a_{2} b\right)=\left\{a_{2}, b\right\} \\
& \operatorname{LR}_{3}=\operatorname{LR}\left(a_{3} b\right)=\left\{a_{3}, b\right\}
\end{aligned}
$$
follows:

The LRN sets of adjacent pair of major vertices are as

$$
\begin{aligned}
& \operatorname{LR}_{4}=\operatorname{LR}\left(a_{1} a_{2}\right)=\left\{a_{1}, a_{2}\right\} \\
& \operatorname{LR}_{5}=\operatorname{LR}\left(a_{2} a_{3}\right)=\left\{a_{2}, a_{3}\right\} \\
& \operatorname{LR}_{6}=\operatorname{LR}\left(a_{3} a_{1}\right)=\left\{a_{1}, a_{3}\right\}
\end{aligned}
$$

From above LRN sets, $\left|\operatorname{LR}\left(e_{i}\right)\right|=2$, where $1 \leq i \leq 6$ and $\left|\bigcup_{i=1}^{6} \operatorname{LR}\left(e_{i}\right)\right|=\left|V\left(J_{3,0}\right)\right|=4$. Hence, a function $\Gamma: V\left(J_{3,0}\right) \longrightarrow[0,1]$ is an LRF defined by $\Gamma(v)=(1 / 2)$ for each $v \in V\left(J_{3,0}\right)$. Therefore, by Proposition 1 ,

$$
\operatorname{dim}_{l f}\left(J_{3,0}\right)=\frac{\left|V\left(J_{3,0}\right)\right|}{2}=2 .
$$

Theorem 5. Let $J_{4,0}$ be a generalized gear network, then

$$
\frac{5}{4} \leq \operatorname{dim}_{l f}\left(J_{4,0}\right) \leq \frac{5}{3} .
$$

Proof. For $m=4$ and $k=0$, the LRN sets are given as follows.

The LRN sets of adjacent pair of major vertices are as follows:

$$
\begin{aligned}
& \operatorname{LR}_{1}=\operatorname{LR}\left(a_{1} a_{2}\right)=\left\{a_{1}, a_{2}, a_{3}, a_{4}\right\} \\
& \operatorname{LR}_{2}=\operatorname{LR}\left(a_{2} a_{3}\right)=\left\{a_{1}, a_{2}, a_{3}, a_{4}\right\} \\
& \operatorname{LR}_{3}=\operatorname{LR}\left(a_{3} a_{4}\right)=\left\{a_{1}, a_{2}, a_{3}, a_{4}\right\} \\
& \operatorname{LR}_{4}=\operatorname{LR}\left(a_{4} a_{5}\right)=\left\{a_{1}, a_{2}, a_{3}, a_{4}\right\}
\end{aligned}
$$

The LRN sets of major vertices with central vertex are as follows:

$$
\begin{aligned}
& \mathrm{LR}_{5}=\operatorname{LR}\left(a_{1} b\right)=\left\{a_{1}, a_{3}, b\right\} \\
& \mathrm{LR}_{6}=\operatorname{LR}\left(a_{2} b\right)=\left\{a_{2}, a_{4}, b\right\} \\
& \mathrm{LR}_{7}=\operatorname{LR}\left(a_{3} b\right)=\left\{a_{1}, a_{3}, b\right\}
\end{aligned}
$$

$$
\operatorname{LR}_{8}=\operatorname{LR}\left(a_{4} b\right)=\left\{a_{2}, a_{4}, b\right\}
$$

From above LRN sets, $\operatorname{LR}\left|\left(a_{i} b\right)\right|=3$, where $1 \leq i \leq 4$ and $\left|\operatorname{LR}\left(a_{i} b\right)\right|<|\operatorname{LR}(x)| \forall x \in E\left(J_{4,0}\right)$. Moreover, $\bigcup_{i=1}^{4} \operatorname{LR}\left(a_{i} b\right)$ $=V\left(J_{4,0}\right)$; this implies $\quad\left|\bigcup_{i=1}^{4} \operatorname{LR}\left(a_{i} b\right)\right|=4$ and $\left|\operatorname{LR}(x) \cap \bigcup_{i=1}^{4} \operatorname{LR}\left(a_{i} b\right)\right|=4$. Therefore, a function $\Gamma: V\left(J_{4,0}\right) \longrightarrow[0,1]$ defined by $\Gamma(v)=(1 / 3)$ for each $v \in V\left(J_{4,0}\right)$ is an upper LRF. Consequently, by Theorem 2, $\operatorname{dim}_{l f}\left(J_{4,0}\right) \leq(5 / 3)$.

From above $\operatorname{LRN}$ sets, $\left|\operatorname{LR}\left(a_{i} a_{i+1}\right)\right|=4$, where $1 \leq i \leq 4$ and $\left|\operatorname{LR}\left(a_{i} a_{i+1}\right)\right| \geq \mid\left(\operatorname{LR}(x) \mid, \quad \forall x \in E\left(J_{4,0}\right)\right.$. Therefore, a function $\Gamma^{\prime}: V\left(J_{4,0}\right) \longrightarrow[0,1]$ is defined by $\Gamma^{\prime}(v)=(1 / 4)$ which is a lower LRF for each $v \in V\left(J_{4,0}\right)$. Hence, by Theorem $3, \operatorname{dim}_{l f}\left(J_{4,0}\right) \geq(5 / 4)$.

Consequently,

$$
\frac{5}{4} \leq \operatorname{dim}_{l f}\left(J_{4,0}\right) \leq \frac{5}{3}
$$

Theorem 6. Let $J_{5,0}$ be the generalized gear network, then

$$
\operatorname{dim}_{l f}\left(J_{5,0}\right)=\frac{3}{2}
$$

Proof. For $m=5$ and $k=0$, the LRN sets are given as follows:

The LRN sets of major vertices with central vertex are as follows:

$$
\begin{aligned}
& \operatorname{LR}_{1}=\operatorname{LR}\left(a_{1} b\right)=\left\{a_{1}, a_{3}, a_{4}, b\right\} \\
& \operatorname{LR}_{2}=\operatorname{LR}\left(a_{2} b\right)=\left\{a_{2}, a_{4}, a_{5}, b\right\} \\
& \operatorname{LR}_{3}=\operatorname{LR}\left(a_{3} b\right)=\left\{a_{3}, a_{1}, a_{5}, b\right\} \\
& \operatorname{LR}_{4}=\operatorname{LR}\left(a_{4} b\right)=\left\{a_{1}, a_{2}, a_{4}, b\right\} \\
& \operatorname{LR}_{5}=\operatorname{LR}\left(a_{5} b\right)=\left\{a_{2}, a_{3}, a_{5}, b\right\}
\end{aligned}
$$

The LRN sets of major vertices with central vertex are as follows:

$$
\begin{aligned}
& \operatorname{LR}_{6}=\operatorname{LR}\left(a_{1} a_{2}\right)=\left\{a_{1}, a_{2}, a_{3}, a_{5}\right\} \\
& \operatorname{LR}_{7}=\operatorname{LR}\left(a_{2} a_{3}\right)=\left\{a_{1}, a_{2}, a_{3}, a_{4}\right\} \\
& \operatorname{LR}_{8}=\operatorname{LR}\left(a_{3} a_{4}\right)=\left\{a_{2}, a_{3}, a_{4}, a_{5}\right\} \\
& \operatorname{LR}_{9}=\operatorname{LR}\left(a_{4} a_{5}\right)=\left\{a_{1}, a_{3}, a_{4}, a_{5}\right\} \\
& \operatorname{LR}_{10}=\operatorname{LR}\left(a_{1} a_{5}\right)=\left\{a_{1}, a_{2}, a_{4}, a_{5}\right\}
\end{aligned}
$$

From above $\operatorname{LRN}$ sets, $\left|\operatorname{LR}\left(e_{i}\right)\right|=4$, where $1 \leq i \leq 10$. Hence, by Corollary 1 ,

$$
\operatorname{dim}_{l f}\left(J_{5,0}\right)=\frac{3}{2}
$$

Theorem 7. Let $J_{m, 0}$ with $m \geq 6$ be a generalized gear network, then

$$
\frac{m+1}{m-1} \leq \operatorname{dim}_{l f}\left(J_{m, 0}\right) \leq \frac{m}{4}
$$

Proof. To prove the result, we have the following cases:

Case 1: for $k=0$ and $m=6$, the LRN sets are given as follows: 
The LRN sets of major vertices with central vertex are as follows:

$$
\begin{aligned}
& \operatorname{LR}_{1}=\operatorname{LR}\left(a_{1} b\right)=\left\{a_{1}, a_{3}, a_{4}, a_{5}, b\right\} \\
& \operatorname{LR}_{2}=\operatorname{LR}\left(a_{2} b\right)=\left\{a_{2}, a_{4}, a_{5}, a_{6}, b\right\} \\
& \operatorname{LR}_{3}=\operatorname{LR}\left(a_{3} b\right)=\left\{a_{3}, a_{5}, a_{6}, a_{1}, b\right\} \\
& \operatorname{LR}_{4}=\operatorname{LR}\left(a_{4} b\right)=\left\{a_{4}, a_{6}, a_{1}, a_{2}, b\right\} \\
& \operatorname{LR}_{5}=\operatorname{LR}\left(a_{5} b\right)=\left\{a_{5}, a_{1}, a_{2}, a_{3}, b\right\} \\
& \operatorname{LR}_{6}=\operatorname{LR}\left(a_{6} b\right)=\left\{a_{6}, a_{2}, a_{3}, a_{4}, b\right\}
\end{aligned}
$$

The LRN sets of adjacent pair of major vertices:

$$
\begin{aligned}
& \operatorname{LR}_{7}=\operatorname{LR}\left(a_{1} a_{2}\right)=\left\{a_{1}, a_{2}, a_{3}, a_{6}\right\} \\
& \operatorname{LR}_{8}=\operatorname{LR}\left(a_{2} a_{3}\right)=\left\{a_{2}, a_{3}, a_{1}, a_{4}\right\} \\
& \operatorname{LR}_{9}=\operatorname{LR}\left(a_{3} a_{4}\right)=\left\{a_{3}, a_{4}, a_{5}, a_{2}\right\} \\
& \operatorname{LR}_{10}=\operatorname{LR}\left(a_{4} a_{5}\right)=\left\{a_{4}, a_{5}, a_{6}, a_{3}\right\} \\
& \operatorname{LR}_{11}=\operatorname{LR}\left(a_{5} a_{6}\right)=\left\{a_{4}, a_{5}, a_{6}, a_{1}\right\} \\
& \operatorname{LR}_{12}=\operatorname{LR}\left(a_{6} a_{1}\right)=\left\{a_{1}, a_{5}, a_{6}, a_{2}\right\}
\end{aligned}
$$

From above LRN sets, $\left|\operatorname{LR}\left(a_{i} a_{i+1}\right)\right|=4$, where $1 \leq i \leq 6$ and $\left|\operatorname{LR}\left(a_{i} a_{i+1}\right)\right| \leq \mid\left(\operatorname{LR}(x) \mid, \forall x \in E\left(J_{4,0}\right)\right.$. Moreover, $\left|\bigcup_{i=1}^{6} \operatorname{LR}\left(a_{i} a_{i+1}\right)\right|=6$ and $\left|\operatorname{LR}(x) \cap \bigcup_{i=1}^{6} \operatorname{LR}\left(a_{i} a_{i+1}\right)\right|$ $\geq|\operatorname{LR}(x)|$. Therefore, a function $\Gamma: V\left(J_{6,0}\right) \longrightarrow[0,1]$ defined as an upper LRF function $\Gamma(x)=(1 / 4)$ for each $x \in \bigcup_{i=1}^{6} \operatorname{LR}\left(a_{i} a_{i+1}\right)$ and 0 otherwise. Therefore, by Theorem 2, $\operatorname{dim}_{l f}\left(J_{6,0}\right) \leq(3 / 2)$.

Since $\left|\operatorname{LR}\left(a_{i} b\right)\right|=5$, where $\quad 1 \leq i \leq 6 \quad$ and $\left|\operatorname{LR}\left(a_{i} b\right)\right| \geq|\operatorname{LR}(x)|, \forall x \in E\left(J_{4,0}\right)$. Therefore, a function $\Gamma^{\prime}: V\left(J_{6,0}\right) \longrightarrow[0,1]$ defined by $\Gamma^{\prime}(v)=(1 / 5)$ for each $v \in V\left(J_{6,0}\right)$ is a lower LRF, Consequently, by Theorem $3, \operatorname{dim}_{l f}\left(J_{6,0}\right) \geq(7 / 5)$.

$$
\frac{7}{5} \leq \operatorname{dim}_{l f}\left(J_{6,0}\right) \leq \frac{3}{2}
$$

Case 2: for $k=0$ and $m \geq 7$.

For $m \geq 7$ : by Lemma $1,\left|\operatorname{LR}\left(e_{i}\right)\right|=\left|\operatorname{LR}\left(a_{i} a_{i+1}\right)\right|=4$ and $\left|\operatorname{LR}(x) \cap \bigcup_{i=1}^{m} \operatorname{LR}\left(a_{i} a_{i+1}\right)\right| \geq\left|\operatorname{LR}\left(a_{i} a_{i+1}\right)\right|, \forall x \in E\left(J_{m, 0}\right)$. Furthermore, $\left|\bigcup_{i=1}^{m} \operatorname{LR}\left(e_{i}\right)\right|=m$. Hence, a function $\Gamma: V\left(J_{m, 0}\right) \longrightarrow[0,1]$ is an upper LRF with minimum cardinality defined as

$$
\Gamma(v)= \begin{cases}\frac{1}{4}, & \text { for } v \in \bigcup_{i=1}^{m} \operatorname{LR}\left(e_{i}\right), \\ 0, & \text { for } v \in V\left(J_{m, 0}\right)-\bigcup_{i=1}^{m} \operatorname{LR}\left(e_{i}\right) .\end{cases}
$$

Consequently, by Theorem $2 \operatorname{dim}_{l f}\left(J_{m, 0}\right) \leq \sum_{i=1}^{m}(1$ /4) $=(\mathrm{m} / 4)$.

By Lemma 1, $\left|\operatorname{LR}\left(a_{i} b\right)\right|=m-1$, where $1 \leq i \leq m$ and $\left|\operatorname{LR}\left(a_{i} b\right)\right| \geq \mid \operatorname{LR}(x), \forall x \in E\left(J_{m, 0}\right)$. Furthermore, $\bigcup_{i=1}^{m}$ $\operatorname{LR}\left(a_{i} b\right)=V\left(J_{m, 0}\right)$; this implies $\left|\bigcup_{i=1}^{m} \operatorname{LR}\left(a_{i} b\right)\right|=$ $m+1$. Hence, function $\gamma: V\left(J_{m, 0}\right) \longrightarrow[0,1]$ is lower LRF defined by $\gamma(v)=(1 /(m-1))$ for each $v \in V\left(J_{m, 0}\right)$; therefore, by Theorem $3, \operatorname{dim}_{l f}\left(J_{m, 0}\right) \geq$ $((m+1) /(m-1))$.

Consequently,

$$
\frac{m+1}{m-1} \leq \operatorname{dim}_{l f}\left(J_{m, 0}\right) \leq \frac{m}{4} .
$$

4.2. For $k=2$ and $m \cong(1 \bmod 2)$

Theorem 8. Let $J_{3,2}$ be a generalized gear network, then $\operatorname{dim}_{l f}\left(J_{3,2}\right)=(5 / 4)$.

Proof. Let $A=\left\{a_{1}, a_{2}, a_{3}\right\}$ be the set of major, $B=\left\{b_{1}^{1}, b_{2}^{1}, b_{1}^{2}, b_{2}^{2}, b_{1}^{3}, b_{1}^{3}, b_{2}^{3}\right\}$ is the set of minor vertices, respectively, and $b$ is the central vertex. For $k=2$ and $m=3$, the LRN sets are given as follows.

The LRN sets of major vertices to central vertex are as follows:

$$
\begin{aligned}
& \operatorname{LR}_{1}=\operatorname{LR}\left(a_{1} b\right)=\left\{a_{1}, a_{2}, a_{3}, b_{1}^{1}, b_{1}^{2}, b_{2}^{2}, b_{2}^{3}, b\right\} \\
& \operatorname{LR}_{2}=\operatorname{LR}\left(a_{2} b\right)=\left\{a_{1}, a_{2}, a_{3}, b_{2}^{1}, b_{1}^{2}, b_{2}^{3}, b_{1}^{3}, b\right\} \\
& \operatorname{LR}_{3}=\operatorname{LR}\left(a_{3} b\right)=\left\{a_{1}, a_{2}, a_{3}, b_{1}^{1}, b_{2}^{1}, b_{2}^{2}, b_{2}^{3}, b\right\}
\end{aligned}
$$

The LRN sets of major to minor vertices are as follows:

$$
\begin{aligned}
& \operatorname{LR}_{4}=\operatorname{LR}\left(a_{1} b_{1}^{1}\right)=\left\{a_{1}, a_{3}, b_{1}^{1}, b_{2}^{1}, b_{2}^{2}, b_{1}^{3}, b_{2}^{3}, b\right\} \\
& \operatorname{LR}_{5}=\operatorname{LR}\left(a_{2} b_{1}^{2}\right)=\left\{a_{1}, a_{2}, b_{1}^{1}, b_{2}^{1}, b_{1}^{2}, b_{2}^{2}, b_{2}^{3}, b\right\} \\
& \operatorname{LR}_{6}=\operatorname{LR}\left(a_{3} b_{1}^{3}\right)=\left\{a_{2}, a_{3}, b_{2}^{1}, b_{1}^{2}, b_{2}^{2}, b_{1}^{3}, b_{2}^{3}, b\right\} \\
& \operatorname{LR}_{7}=\operatorname{LR}\left(a_{1} b_{2}^{3}\right)=\left\{a_{1}, a_{2}, b_{1}^{1}, b_{2}^{1}, b_{1}^{2}, b_{1}^{3}, b_{2}^{3}, b\right\} \\
& \operatorname{LR}_{8}=\operatorname{LR}\left(a_{2} b_{2}^{1}\right)=\left\{a_{2}, a_{3}, b_{1}^{1}, b_{2}^{1}, b_{1}^{2}, b_{2}^{2}, b_{1}^{3}, b\right\} \\
& \operatorname{LR}_{9}=\operatorname{LR}\left(a_{3} b_{2}^{2}\right)=\left\{a_{1}, a_{3}, b_{1}^{1}, b_{1}^{2}, b_{2}^{2}, b_{2}^{3}, b_{1}^{3}, b\right\}
\end{aligned}
$$

The LRN sets of adjacent pair of minor vertices are as follows:

$$
\begin{aligned}
& \operatorname{LR}_{10}=\operatorname{LR}\left(b_{1}^{1} b_{2}^{1}\right)=\left\{a_{1}, a_{2}, b_{1}^{1}, b_{2}^{1}, b_{1}^{2}, b_{2}^{2}, b_{1}^{3}, b_{2}^{3}\right\} \\
& \operatorname{LR}_{11}=\operatorname{LR}\left(b_{1}^{2} b_{2}^{2}\right)=\left\{a_{2}, a_{3}, b_{1}^{1}, b_{2}^{1}, b_{1}^{2}, b_{2}^{2}, b_{1}^{3}, b_{2}^{3}\right\} \\
& \operatorname{LR}_{12}=\operatorname{LR}\left(b_{1}^{3} b_{2}^{3}\right)=\left\{a_{1}, a_{3}, b_{1}^{1}, b_{2}^{1}, b_{1}^{2}, b_{2}^{2}, b_{1}^{3}, b_{2}^{3}\right\}
\end{aligned}
$$

Since each $\left|\operatorname{LR}\left(e_{i}\right)\right|=8$, where $1 \leq i \leq 12$, by Corollary 1 , $\operatorname{dim}_{l f}\left(J_{3,2}\right)=\left(\left|V\left(J_{3,2}\right)\right| / \alpha\right)=(5 / 4)$.

Theorem 9. Let $J_{m, 2}$, where $m \cong(1 \bmod 2)$, be a generalized gear network. Then, for $1 \leq i \leq m$,

$$
\frac{3 m+1}{3 m-1} \leq \operatorname{dim}_{l f}\left(J_{m, 2}\right) \leq \frac{3 m}{8} .
$$

Proof. To prove the result, we have the following cases:

Case 1: for, $k=2$ and $m=5$, the LRN sets are given as follows.

The LRN sets of major vertices with central vertex are as follows:

$$
\begin{aligned}
& \operatorname{LR}_{1}=\operatorname{LR}\left(a_{1} b\right)=V\left(J_{2,5}\right)-\left\{b_{2}^{2}, b_{1}^{5}\right\} \\
& \operatorname{LR}_{2}=\operatorname{LR}\left(a_{2} b\right)=V\left(J_{2,5}\right)-\left\{b_{2}^{1}, b_{2}^{2}\right\} \\
& \mathrm{LR}_{3}=\operatorname{LR}\left(a_{3} b\right)=V\left(J_{2,5}\right)-\left\{b_{2}^{2}, b_{2}^{3}\right\} \\
& \mathrm{LR}_{4}=\operatorname{LR}\left(a_{4} b\right)=V\left(J_{2,5}\right)-\left\{b_{1}^{3}, b_{2}^{4}\right\} \\
& \mathrm{LR}_{5}=\operatorname{LR}\left(a_{4} b\right)=V\left(J_{2,5}\right)-\left\{b_{2}^{4}, b_{2}^{5}\right\}
\end{aligned}
$$

The LRN sets of adjacent pair of minor vertices are as follows:

$$
\begin{aligned}
& \mathrm{LR}_{6}=\operatorname{LR}\left(a_{1} b_{1}^{1}\right)==V\left(J_{2,5}\right)-\left\{a_{2}, b_{1}^{2}\right\} \\
& \operatorname{LR}_{7}=\operatorname{LR}\left(a_{2} b_{1}^{2}\right)==V\left(J_{2,5}\right)-\left\{a_{3}, b_{1}^{3}\right\}
\end{aligned}
$$




$$
\begin{aligned}
\operatorname{LR}_{8}=\operatorname{LR}\left(a_{3} b_{1}^{3}\right) & ==V\left(J_{2,5}\right)-\left\{a_{4}, b_{1}^{4}\right\} \\
\operatorname{LR}_{9} & =\operatorname{LR}\left(a_{4} b_{1}^{4}\right)==V\left(J_{2,5}\right)-\left\{a_{5}, b_{1}^{5}\right\} \\
\operatorname{LR}_{10}=\operatorname{LR}\left(a_{5} b_{1}^{5}\right) & ==V\left(J_{2,5}\right)-\left\{a_{1}, b_{1}^{1}\right\} \\
\operatorname{LR}_{11}=\operatorname{LR}\left(a_{2} b_{2}^{1}\right) & ==V\left(J_{2,5}\right)-\left\{a_{1}, b_{2}^{5}\right\} \\
\operatorname{LR}_{12}=\operatorname{LR}\left(a_{3} b_{2}^{2}\right) & ==V\left(J_{2,5}\right)-\left\{a_{2}, b_{2}^{2}\right\} \\
\operatorname{LR}_{13}=\operatorname{LR}\left(a_{4} b_{2}^{3}\right) & ==V\left(J_{2,5}\right)-\left\{a_{3}, b_{2}^{2}\right\} \\
\operatorname{LR}_{14} & =\operatorname{LR}\left(a_{5} b_{2}^{4}\right)==V\left(J_{2,5}\right)-\left\{a_{4}, b_{2}^{3}\right\} \\
\operatorname{LR}_{15}=\operatorname{LR}\left(a_{1} b_{2}^{5}\right) & ==V\left(J_{2,5}\right)-\left\{a_{5}, b_{2}^{4}\right\}
\end{aligned}
$$

The LRN sets of adjacent pair of minor vertices are as follows:

$$
\begin{aligned}
& \operatorname{LR}_{16}=\operatorname{LR}\left(b_{1}^{1} b_{2}^{1}\right)=\left\{a_{1}, a_{2}, b_{1}^{1}, b_{2}^{1}, b_{1}^{2}, b_{2}^{2}, b_{1}^{3}, b_{2}^{3}\right\} \\
& \operatorname{LR}_{17}=\operatorname{LR}\left(b_{1}^{2} b_{2}^{2}\right)=\left\{a_{2}, a_{3}, b_{1}^{1}, b_{2}^{1}, b_{1}^{2}, b_{2}^{2}, b_{1}^{3}, b_{2}^{3}\right\} \\
& \operatorname{LR}_{18}=\operatorname{LR}\left(b_{1}^{3} b_{2}^{3}\right)=\left\{a_{1}, a_{3}, b_{1}^{2}, b_{2}^{2}, b_{1}^{5}, b_{2}^{5}, b_{1}^{4}, b_{2}^{4}\right\} \\
& \operatorname{LR}_{19}=\operatorname{LR}\left(b_{1}^{4} b_{2}^{4}\right)=\left\{a_{1}, a_{3}, b_{1}^{1}, b_{2}^{1}, b_{1}^{3}, b_{2}^{3}, b_{1}^{5}, b_{2}^{5}\right\} \\
& \operatorname{LR}_{20}=\operatorname{LR}\left(b_{1}^{5} b_{2}^{5}\right)=\left\{a_{1}, a_{3}, b_{1}^{1}, b_{2}^{1}, b_{1}^{3}, b_{2}^{3}, b_{1}^{4}, b_{2}^{4}\right\}
\end{aligned}
$$

From above LRN sets, $\left|\operatorname{LR}\left(e_{i}\right)\right|=\left|\operatorname{LR}\left(b_{i}^{i} b_{i+1}^{i}\right)\right|=8$, where $1 \leq i \leq 5$ and $\left|\operatorname{LR}\left(e_{i}\right)\right| \leq|\operatorname{LR}(x)| \forall x \in E\left(J_{5,2}\right)$. Furthermore, $\bigcup_{i=1}^{5} \operatorname{LR}\left(e_{i}\right)=15$ and $\mid \operatorname{LR}(x) \cap \bigcup_{i=1}^{5} \operatorname{LR}$ $\left(e_{i}\right)|\geq| \operatorname{LR}\left(e_{i}\right) \mid=8$. Hence, a function $\gamma_{5} V\left(J_{5,2}\right)$ $\longrightarrow[0,1]$ defined by $\gamma(v)=(1 / 8)$, if $v \in \bigcup_{i=1}^{5} \operatorname{LR}\left(e_{i}\right)$ and 0 otherwise is an upper LRF. Consequently, by Theorem 2, $\operatorname{dim}_{l f}\left(J_{5,2}\right) \leq(15 / 8)$.

From above LRN sets, $\left|\operatorname{LR}\left(e_{i}^{\prime}\right)\right|=14$, where $1 \leq i \leq 15$ and $\left|\operatorname{LR}\left(e_{i}^{\prime}\right)\right| \geq \operatorname{LR}(x) \forall x \in E\left(J_{5,2}\right)$. Therefore, there exists a lower LRF A function $\gamma^{\prime}: V\left(J_{5,2}\right) \longrightarrow[0,1]$ defined by $\gamma^{\prime}(v)=(1 / 12)$ is an LRF of maximum cardinality for each $v \in V\left(J_{5,2}\right)$. Hence, by Theorem 3 , $\operatorname{dim}_{l f}\left(J_{5,2}\right) \geq(8 / 7)$.

Consequently,

$$
\frac{8}{7} \leq \operatorname{dim}_{l f}\left(J_{5,2}\right) \leq \frac{15}{8} .
$$

Case 2: for $k=2, \quad m \geq 5$, and $m \cong 1(\bmod 2)$, $\operatorname{LR}\left(e_{i}\right)=\operatorname{LR}\left(b_{k / 2}^{i} b_{(k / 2)+1}^{i}\right)$, where $1 \leq k \leq n$. By Lemma 2, we have the following:

(i) $\left|\operatorname{LR}\left(e_{i}\right)\right|=\left|\operatorname{LR}\left(b_{k / 2}^{i} b_{(k / 2)+1}^{i}\right)\right|=8$

(ii) $\left|\operatorname{LR}(x) \cap \bigcup_{i=1}^{n} \operatorname{LR}\left(e_{i}\right)\right| \geq 8 \forall x \in E\left(J_{m, 2}\right)$

Therefore, a function $\Gamma: V\left(J_{m, 2}\right) \longrightarrow[0,1]$ is an upper LRF with minimum cardinality defined by

$$
\Gamma(v)= \begin{cases}\frac{1}{8}, & \text { if } v \in \bigcup_{i=1}^{m} \operatorname{LR}\left(e_{i}\right), \\ 0, & \text { if } v \in V\left(J_{m, 2}\right)-\bigcup_{i=1}^{m} \operatorname{LR}\left(e_{i}\right) .\end{cases}
$$

Consequently, by Theorem $2, \operatorname{dim}_{l f}\left(J_{m, 2}\right) \leq(3 m / 8)$. Since $\left|\operatorname{LR}\left(a_{i} b_{i}\right)\right|=\left|V\left(J_{m, 2}\right)\right|-2$ and $\operatorname{LR}\left|\left(a_{i} b_{i}\right)\right| \geq \operatorname{LR}(x)$, $\forall x \in E\left(J_{m, 2}\right)$. Hence, the function $\Gamma^{\prime}: V\left(J_{m, 2}\right)$ $\longrightarrow[0,1]$ is a lower LRF with maximum cardinality, defined by $\Gamma^{\prime}(v)=(1 /(3 m-1)) \forall v \in V \quad\left(J_{m, 2}\right)$. Therefore, by Theorem 3, $\operatorname{dim}_{l f}\left(J_{m, 2}\right) \geq$ $((3 m+1) /(3 m-1))$.

Consequently,

$$
\frac{3 m+1}{3 m-1} \leq \operatorname{dim}_{l f}\left(J_{m, 2}\right) \leq \frac{3 m}{8} .
$$

Theorem 10. Let $J_{m, k}$ be a generalized gear network with $m \geq 4, k \geq 2$, where $k \cong 0(\bmod 2)$. Then,

$$
\frac{m(k+1)+1}{m(k+1)} \leq \operatorname{dim}_{l f}\left(J_{m, k}\right) \leq \frac{m(k+1)}{2 k+4} .
$$

Proof. To prove the result, we have the following cases:

Case 1: for, $m=4$ and $k=2$, the LRN sets are given as follows.

Adjacent pair of minor vertices is as follows:

$$
\begin{aligned}
& \operatorname{LR}_{1}=\operatorname{LR}\left(b_{1}^{1} b_{2}^{1}\right)=V\left(J_{4,2}\right)-\left\{a_{3}, b_{1}^{3}, b_{2}^{3}, a_{4}, b\right\} \\
& \operatorname{LR}_{2}=\operatorname{LR}\left(b_{1}^{2} b_{2}^{2}\right)=V\left(J_{4,2}\right)-\left\{b_{1}^{4}, a_{1}, b, b_{2}^{4}, b_{1}^{4}\right\} \\
& \operatorname{LR}_{3}=\operatorname{LR}\left(b_{1}^{3} b_{2}^{3}\right)=V\left(J_{4,2}\right)-\left\{a_{1}, b_{1}^{1}, b_{2}^{1}, a_{2}, b\right\} \\
& \operatorname{LR}_{4}=\operatorname{LR}\left(b_{1}^{4} b_{2}^{4}\right)=V\left(J_{4,2}\right)-\left\{b_{1}^{2}, a_{2}, b, a_{3}, b_{2}^{2}\right\}
\end{aligned}
$$

Major with central vertex.

$$
\begin{aligned}
& \operatorname{LR}_{5}=\operatorname{LR}\left(a_{1} b\right)=V\left(J_{4,2}\right)-\left\{b_{2}^{1}, b_{1}^{4}\right\} \\
& \operatorname{LR}_{6}=\operatorname{LR}\left(a_{2} b\right)=V\left(J_{4,2}\right)-\left\{b_{1}^{1}, b_{2}^{2}\right\} \\
& \operatorname{LR}_{7}=\operatorname{LR}\left(a_{3} b\right)=V\left(J_{4,2}\right)-\left\{b_{2}^{3}, b_{1}^{2}\right\} \\
& \operatorname{LR}_{8}=\operatorname{LR}\left(a_{4} b\right)=V\left(J_{4,2}\right)-\left\{b_{2}^{4}, b_{2}^{1}\right\}
\end{aligned}
$$

Major with minor vertex.

$$
\begin{aligned}
& \operatorname{LR}_{9}=\operatorname{LR}\left(a_{1} b_{1}^{1}\right)=V\left(J_{4,2}\right)-\left\{a_{2}\right\} \\
& \operatorname{LR}_{10}=\operatorname{LR}\left(a_{1} b_{2}^{4}\right)=V\left(J_{4,2}\right)-\left\{a_{4}\right\} \\
& \operatorname{LR}_{11}=\operatorname{LR}\left(a_{2} b_{2}^{1}\right)=V\left(J_{4,2}\right)-\left\{a_{1}\right\} \\
& \operatorname{LR}_{12}=\operatorname{LR}\left(a_{2} b_{1}^{2}\right)=V\left(J_{4,2}\right)-\left\{a_{3}\right\} \\
& \operatorname{LR}_{13}=\operatorname{LR}\left(a_{4} b_{2}^{3}\right)=V\left(J_{4,2}\right)-\left\{a_{3}\right\} \\
& \operatorname{LR}_{14}=\operatorname{LR}\left(a_{4} b_{2}^{3}\right)=V\left(J_{4,2}\right)-\left\{a_{3}\right\} \\
& \operatorname{LR}_{15}=\operatorname{LR}\left(a_{4} b_{1}^{4}\right)=V\left(J_{4,2}\right)-\left\{a_{1}\right\}
\end{aligned}
$$

From above $\operatorname{LRN}$ sets, $\left|\operatorname{LR}\left(e_{i}\right)\right|=\left|\operatorname{LR}\left(b_{1}^{i} b_{2}^{i}\right)\right|=8$, where $1 \leq i \leq 4$ and $\left|\operatorname{LR}\left(e_{i}\right)\right| \leq|\operatorname{LR}(x)| \forall x \in E\left(J_{4,2}\right)$. Furthermore, $\left|\bigcup_{i=1}^{4} \operatorname{LR}\left(e_{i}\right)\right|=12$ and $\mid \operatorname{LR}(x) \cap \bigcup_{i=1}^{4} \operatorname{LR}$ $\left(e_{i}\right)|\geq| \operatorname{LR}\left(e_{i}\right) \mid=8$. Hence, a function $\gamma: V\left(J_{4,2}\right)$ $\longrightarrow[0,1]$ defined by $\gamma(v)=(1 / 8)$ if $v \in \bigcup_{i=1}^{4} \operatorname{LR}\left(e_{i}\right)$ and 0 otherwise is a lower LRF with minimum cardinality. Consequently, by Theorem 2, $\operatorname{dim}_{l f}\left(J_{4,2}\right) \leq(3 / 2)$.

From above LRN sets, $\left|\operatorname{LR}\left(e_{i}^{\prime}\right)\right|=12$, where $1 \leq i \leq 6$ and $\left|\operatorname{LR}\left(e_{i}^{\prime}\right)\right| \geq \operatorname{LR}(x) \forall x \in E\left(J_{4,2}\right)$. Therefore, a function $\gamma^{\prime}: V\left(J_{4,2}\right) \longrightarrow[0,1]$ defined by $\gamma^{\prime}(v)=(1 / 12)$ is a lower LRF with maximum cardinality for each $v \in V\left(J_{4,2}\right)$.

$$
\frac{13}{12} \leq \operatorname{dim}_{l f}\left(J_{4,2}\right) \leq \frac{3}{2}
$$

Case 2: for $k=4$ and $m=3$, the possible LRN sets are as follows.

Central to major vertex.

$$
\begin{aligned}
& \operatorname{LR}_{1}\left(a_{1} b\right)=V\left(J_{3,4}\right)-\left\{b_{3}^{1}, b_{2}^{3}\right\} \\
& \operatorname{LR}_{2}\left(a_{2} b\right)=V\left(J_{3,4}\right)-\left\{b_{3}^{2}, b_{2}^{1}\right\} \\
& \operatorname{LR}_{3}\left(a_{3} b\right)=V\left(J_{3,4}\right)-\left\{b_{3}^{3}, b_{2}^{3}\right\}
\end{aligned}
$$

Major with minor vertex. 
TABLE 3: Unboundedness of LFMDs.

\begin{tabular}{lccr}
\hline Network & LFMDs & $\begin{array}{c}\text { Lower bound of } \\
\text { LF-metric dimension } \\
\text { when } m \longrightarrow \infty\end{array}$ & $\begin{array}{c}\text { Upper bound of } \\
\text { LF-metric dimension } \\
\text { when } m \longrightarrow \infty\end{array}$ \\
\hline$J_{(m, 0)}$ & $((m+1) /(m-1)) \leq \operatorname{dim}_{l f}\left(J_{m, 0}\right) \leq(m / 4)$ & 1 & $\infty$ \\
$J_{(m, 2)}$ & $((3 m+1) /(3 m-1)) \leq \operatorname{dim}_{l f}\left(J_{m, 2}\right) \leq(3 m / 8)$ & 1 & Unbounded \\
$J_{(m, k)}$ & $((m(k+1)+1) / m(k+1)) \leq \operatorname{dim}_{l f}\left(J_{m, k}\right) \leq(m(k+1) /(2 k+4))$ & 1 & Unbounded \\
\hline
\end{tabular}

$$
\begin{aligned}
& \operatorname{LR}_{4}\left(a_{1} b_{1}^{1}\right)=V\left(J_{3,4}\right)-\left\{b_{4}^{1}\right\} \\
& \operatorname{LR}_{5}\left(a_{2} b_{1}^{2}\right)=V\left(J_{3,4}\right)-\left\{b_{4}^{2}\right\} \\
& \operatorname{LR}_{6}\left(a_{3} b_{1}^{3}\right)=V\left(J_{3,4}\right)-\left\{b_{4}^{3}\right\} \\
& \operatorname{LR}_{7}\left(a_{1} b_{4}^{3}\right)=V\left(J_{3,4}\right)-\left\{b_{1}^{3}\right\} \\
& \operatorname{LR}_{8}\left(a_{2} b_{4}^{1}\right)=V\left(J_{3,4}\right)-\left\{b_{1}^{1}\right\} \\
& \operatorname{LR}_{9}\left(a_{3} b_{4}^{2}\right)=V\left(J_{3,4}\right)-\left\{b_{1}^{2}\right\}
\end{aligned}
$$

Adjacent pair of internal vertices.

$$
\begin{aligned}
& \operatorname{LR}_{10}\left(b_{1}^{1} b_{2}^{1}\right)=V\left(J_{3,4}\right)-\left\{a_{2}, b_{1}^{2}, b_{2}^{2}\right\} \\
& \operatorname{LR}_{11}\left(b_{3}^{1} b_{4}^{1}\right)=V\left(J_{3,4}\right)-\left\{a_{1}, b_{3}^{3}, b_{4}^{3}\right\} \\
& \operatorname{LR}_{12}\left(b_{1}^{2} b_{2}^{2}\right)=V\left(J_{3,4}\right)-\left\{a_{3}, b_{1}^{3}, b_{2}^{3}\right\} \\
& \operatorname{LR}_{13}\left(b_{3}^{2} b_{4}^{2}\right)=V\left(J_{3,4}\right)-\left\{a_{2}, b_{4}^{1}, b_{3}^{1}\right\} \\
& \operatorname{LR}_{14}\left(b_{1}^{3} b_{2}^{3}\right)=V\left(J_{3,4}\right)-\left\{a_{1}, b_{1}^{1}, b_{2}^{1}\right\} \\
& \operatorname{LR}_{15}\left(b_{2}^{3} b_{3}^{3}\right)=V\left(J_{3,4}^{3}\right)-\left\{a_{2}, b_{1}^{2}, b_{4}^{1}\right\} \\
& \operatorname{LR}_{16}\left(b_{3}^{3} b_{4}^{3}\right)=V\left(J_{3,4}^{3}\right)-\left\{a_{3}, b_{4}^{2}, b_{3}^{2}\right\}
\end{aligned}
$$

LRN sets with minimum cardinality are as follows:

$$
\begin{aligned}
& \operatorname{LR}_{17}\left(b_{2}^{1} b_{3}^{1}\right)=V\left(J_{3,4}\right)-\left\{b, b_{4}^{2}, b_{1}^{3}, a_{3}\right\} \\
& \operatorname{LR}_{18}\left(b_{2}^{2} b_{3}^{2}\right)=V\left(J_{3,4}\right)-\left\{b, b_{4}^{3}, b_{1}^{1}, a_{1}\right\} \\
& \operatorname{LR}_{19}\left(b_{2}^{3} b_{3}^{3}\right)=V\left(J_{3,4}\right)-\left\{b, b_{1}^{2}, b_{4}^{1}, a_{2}\right\}
\end{aligned}
$$

From above LRN sets, $\left|\operatorname{LR}\left(e_{i}\right)\right|=\left|\operatorname{LR}\left(b_{2}^{i} b_{3}^{i}\right)\right|=12$, where $1 \leq i \leq 3$ and $\left|\operatorname{LR}\left(e_{i}\right)\right| \leq|\operatorname{LR}(x)| \forall x \in E\left(J_{3,4}\right)$. Moreover, $\bigcup_{i=1}^{3} \operatorname{LR}\left(e_{i}\right)=\left|V\left(J_{3,4}\right)\right|-1$, and this implies $\left|\bigcup_{i=1}^{3} \operatorname{LR}\left(e_{i}\right)\right|=15$ and $\left|\operatorname{LR}(x) \cap \bigcup_{i=1}^{12} \operatorname{LR}\left(e_{i}\right)\right| \geq \mid \operatorname{LR}$ $\left(e_{i}\right) \mid=12$. Hence, a function $\Gamma: V\left(J_{3,4}\right) \longrightarrow[0,1]$ is defined by $\Gamma(v)=(1 / 12)$ which is an upper LRF with minimum cardinality for all $v \in V\left(J_{3,4}\right)$ and 0 otherwise. Therefore, $\operatorname{dim}_{l f}\left(J_{3,4}\right) \leq(5 / 4)$.

From above $\operatorname{LRN}$ sets $\left|\operatorname{LR}\left(e_{i}\right)\right|=6$, where $1 \leq i \leq 6$ and $\left|\operatorname{LR}\left(e_{i}\right)\right| \geq \operatorname{LR}(x), \forall x \in E\left(J_{3,4}\right)$. Therefore, a function $\Gamma^{\prime}: V\left(J_{3,4}\right) \longrightarrow[0,1]$ is defined by $\Gamma^{\prime}(v)=(1 / 6)$ $\forall v \in V\left(J_{3,4}\right)$; hence, by Theorem $3, \operatorname{dim}_{l f}\left(J_{3,4}\right) \geq(4 / 3)$. Consequently,

$$
\frac{4}{3} \leq \operatorname{dim}_{l f}\left(J_{3,4}\right) \leq \frac{8}{5}
$$

Case 3: for $k \cong 0(\bmod 2)$ and $m \geq 4$, the possible LRN sets are $\operatorname{LR}\left(b_{k / 2}^{i} b_{(k / 2)+1}^{i}\right)$, where $1 \leq k \leq n$. By Lemma 2 and 3 , we have
(i) $\left|\operatorname{LR}\left(e_{i}\right)\right|=\left|\operatorname{LR}\left(b_{k / 2}^{i} b_{(k / 2)+1}^{i}\right)\right|=2 k+4$ and $\left|\operatorname{LR}\left(e_{i}\right)\right|$ $\leq|\operatorname{LR}(x)| \forall x \in E\left(J_{m, k}\right)$

(ii) $\left|\operatorname{LR}(x) \cap \bigcup_{i=1}^{m} \operatorname{LR}\left(e_{i}\right)\right| \geq\left|\operatorname{LR}\left(e_{i}\right)\right| \forall x \in E\left(J_{m, k}\right)$

Therefore, $\Gamma: V\left(J_{m, k}\right) \longrightarrow[0,1]$ is an upper LRF defined by

$$
\Gamma(v)= \begin{cases}\frac{1}{2 k+4}, & \text { if } v \in \bigcup_{i=1}^{m} \operatorname{LR}\left(e_{i}\right) \\ 0, & \text { if } v \in V\left(J_{m, k}\right)-\bigcup_{i=1}^{m} \operatorname{LR}\left(e_{i}\right) .\end{cases}
$$

Consequently, by Theorem 2, $\operatorname{dim}_{l f}\left(J_{m, k}\right) \leq$ $(m(k+1) /(2 k+4))$.

Case 4: since $\left|\operatorname{LR}\left(a_{i} b_{i}\right)\right|=\left|V\left(J_{m, k}\right)\right|-1 \quad$ and $\operatorname{LR}\left|\left(a_{i} b_{i}\right)\right| \geq|\operatorname{LR}(x)|, \quad \forall x \in E\left(J_{m, k}\right)$. Therefore, $\Gamma^{\prime}: V$ $\left(J_{m, k}\right) \longrightarrow[0,1] \quad$ defined by $\Gamma^{\prime}(v)=(1 \quad \operatorname{lm}(k$ $+1)), \forall v \in V\left(J_{m, k}\right)$ is lower LRF. Hence, by Theorem 3, $\left.\operatorname{dim}_{l f}\left(J_{m, k}\right)\right) \geq((m(k+1)+1) / m(k+1))$.

Consequently,

$$
\frac{m(k+1)+1}{m(k+1)} \leq \operatorname{dim}_{l f}\left(J_{m, k}\right) \leq \frac{m(k+1)}{2 k+4} .
$$

\subsection{For $k \cong 1(\bmod 2)$ and $m \geq 3$}

Theorem 11. For $k \geq 3, k k \cong 1(\bmod 2)$ and $G \cong J_{m, k}$ is a generalized gear network, and then $\operatorname{dim}_{l f}(G)=1$.
Proof. As there is no cycle of odd length in $G \cong J_{m, k}$, where $k \cong 1(\bmod 2)$. Therefore, $G$ is bipartite network; hence, by Theorem 1, $\operatorname{dim}_{l f}(G)=1$.

\section{Conclusion}

In this article, we studied the LF-metric dimensions of generalized gear networks and established the sharp lower and upper bounds of LF-metric dimensions and computed the exact values in some cases as well.

Exact values of LF-metric dimensions of some cases of the generalized gear networks are as follows:

$$
J_{(3,0)}=2, J_{(5,0)}=(3 / 2) \text { and } J_{(3,2)}=(5 / 4)
$$

Unboundedness is illustrated in Table 3 
Now, this section is closed by raising the following open problem:

Investigate the LF-metric dimension of the nonregular networks such as convex polytopes, Toeplitz, and Prism-related networks.

\section{Data Availability}

The data used to support the findings of this study are included within this article. However, the reader may contact the corresponding author for more details on the data.

\section{Conflicts of Interest}

The authors declare no conflicts of interest regarding this article.

\section{References}

[1] P. J. Slater, "Leaves of trees," Congressus Numerantium, vol. 14, no. 1, pp. 549-559, 1975.

[2] F. Harary and R. Melter, "On the metric dimension of a graph,” Ars Combinatoria, vol. 2, pp. 19-195, 1976.

[3] R. A. Melter and I. Tomescu, "Metric bases in digital geometry," Computer Vision, Graphics, and Image Processing, vol. 25, no. 1, pp. 113-121, 1984.

[4] P. J. Slater, "Dominating and reference sets in a graph," Journal of Mathematical and Physical Sciences, vol. 22, no. 4, pp. 445-455, 1988.

[5] M. R. Garey and D. S. Johnson, Computers and Intractability: A Guide to the Theory of NP-Completeness, Freeman, New York, NY, USA, 1979.

[6] A. Kelenca, N. Tratnik, and I. G. Yero, "Uniquely identifying the edges of a graph: the edge metric dimension," Discrete Applied Mathematics, vol. 251, pp. 204-220, 2018.

[7] G. Chattrand, M. Raines, P. Zhang, and Kalamzoo, "The directed distance dimension of orianted graphs," Mathematica Bhomecia, vol. 125, no. 2, pp. 155-168, 2000.

[8] Z. S. Mufti, M. F. Nadeem, A. Ahmad, and Z. Ahmad, "Computation of edge metric dimension of barycentric subdivision of cayley graphs," Italian Journal of pure and applied Mathematics, vol. 44, pp. 714-722, 2020.

[9] M. Ali, G. Ali, M. Imran, A. Q. Baig, and M. K. Shariq, "On the metric dimension of Mobius ladders," Ars Combinatoria, vol. 105, pp. 403-410, 2012.

[10] S. W. Saputro, R. Simanjuntak, S. Uttunggadewa et al., "The metric dimension of the lexicographic product of graphs," Discrete Mathematics, vol. 313, no. 9, pp. 1045-1051, 2013.

[11] J.-B. Liu, M. F. Nadeem, H. M. A. Siddiqui, and W. Nazir, "Computing metric dimension of certain families of toeplitz graphs," IEEE Access, vol. 7, pp. 126734-126741, 2019.

[12] S. Khuller, B. Raghavachari, and A. Rosenfeld, "Localization in graphs,” Tech. Rep. UMIACS-TR-94-92, Univ. Maryland, College Park, MD, USA, 1994.

[13] Y. Shang, "Percolation in a hierarchical lattice," Zeitschrift Natur-Forschung, vol. 67, no. 5, 2012.

[14] G. Chartrand, L. Eroh, M. A. Johnson, and O. R. Oellermann, "Resolvability in graphs and the metric dimension of a graph," Discrete Applied Mathematics, vol. 105, no. 1-3, pp. 99-113, 2000.

[15] I. Tomescu and I. Javaid, "On the metric dimension of the Jahangir graph," Bulletin Mathématiques de la Societé des
Sciences Mathématiques de Roumanie, vol. 50, pp. 371-376, 2007.

[16] S. Imran, M. Siddiqui, M. Imran et al., "Computing the metric dimension of gear graphs," Symmetry, vol. 10, no. 6, p. 209, 2018.

[17] J. Currie and O. R. Oellermann, "The metric dimension and metric independence of a graph," Journal of Combinatorial Mathematics and Combinatorial Computing, vol. 39, pp. 157-167, 2001.

[18] M. Fehr, S. Gosselin, and O. R. Oellermann, "The metric dimension of cayley digraphs," Discrete Mathematics, vol. 306, no. 1, pp. 31-41, 2006.

[19] S. Arumugam and V. Mathew, "The fractional metric dimension of graphs," Discrete Mathematics, vol. 312, no. 9, pp. 1584-1590, 2012.

[20] M. Feng, B. Lv, and K. Wang, "On the fractional metric dimension of graphs," Discrete Applied Mathematics, vol. 170, pp. 55-63, 2014.

[21] S. W. Saputro, A. Semani, M. Băca, and M. Lascs' akov́, "On fractional metric dimension of comb product graphs," Statistics, Optimization \& Information Computing, vol. 6, pp. 150-158, 2018.

[22] M. Feng and K. Wang, "On the metric dimension and fractional metric dimension for hierarchical product of graphs," Applicable Analysis and Discrete Mathematics, vol. 7, no. 2, pp. 302-313, 2013.

[23] D. A. Krismanto and S. W. Saputro, "Fractional metric dimension of tree and unicyclic graph," Procedia Computer Science, vol. 74, pp. 47-52, 2015.

[24] E. Yi, "The fractional metric dimension of permutation graphs," Acta Mathematica Sinica, English Series, vol. 31, pp. 367-382, 2015.

[25] M. Raza, D. Alrowalili, M. Javaid, and K. Shabbir, "Computing bounds of fractional metric dimension of metal organic graphs," Journal of Chemistry, vol. 2021, 12 pages, 2021.

[26] J. B. Liu, A. Kashif, T. Rashid, and M. Javaid, "Fractional metric dimension of generalized Jahangir graph," Mathematics, vol. 7, pp. 1-10, 2019.

[27] S. Aisyah, M. Utoyo, and L. Susilowati, "On the local fractional metric dimension of corona product networks," IOP Conference, Earth Environ. Sci. Hungarica, vol. 243, pp. 1-4, 2019.

[28] J.-B. Liu, M. K. Aslam, and M. Javaid, "Local fractional metric dimensions of rotationally symmetric and planar networks," IEEE Access, vol. 8, pp. 82404-82420, 2020.

[29] M. Javaid, M. Raza, P. Kumam, and J.-B. Liu, "Sharp bounds of local fractional metric dimensions of connected networks," IEEE Access, vol. 8, pp. 172329-172342, 2020.

[30] M. Javaid, H. Zafar, Q. Zhu, and A. M. Alanazi, "Improved lower bound of LFMD with applications of prism-related networks," Mathematical Problems in Engineering, vol. 2021, Article ID 9950310, 9 pages, 2021.

[31] D. B. West, Introduction to Graph Theory, Prentice Hall, 2 edition, Hoboken, NJ, USA, 2011. 